\title{
NAIR-TENENBAUM BOUNDS UNIFORM WITH RESPECT TO THE DISCRIMINANT
}

\author{
KEVIN HENRIOT
}

\begin{abstract}
For a suitable arithmetic function $F$ and polynomials $Q_{1}, \ldots, Q_{k}$ in $\mathbb{Z}[X]$, Nair and Tenenbaum obtained an upper bound on the short sum $\sum_{x<n \leqslant x+y} F\left(\left|Q_{1}(n)\right|, \ldots,\left|Q_{k}(n)\right|\right)$ with an implicit dependency on the discriminant of $Q_{1} \cdots Q_{k}$. We obtain a similar upper bound uniform in the discriminant.
\end{abstract}

\section{INTRODUCTION}

Let $\mathcal{M}$ denote the class of multiplicative functions $f$ such that

(1) there exists $A \geqslant 1$ such that $f\left(p^{\ell}\right) \leqslant A^{\ell}$ for any prime $p$ and any $\ell \in \mathbb{N}$,

(2) for all $\varepsilon>0$ there exists $B=B(\varepsilon)>0$ such that $f(n) \leqslant B n^{\varepsilon}$ for any $n \in \mathbb{N}$.

Let also $\alpha, \beta \in] 0,1[$. For $f \in \mathcal{M}$ and $(a, q)=1$, Shiu [12] proved that

$$
\sum_{\substack{x<n \leqslant x+y \\ n \equiv a \bmod q}} f(n) \ll \frac{y}{\varphi(q)} \frac{1}{\log x} \exp \left(\sum_{\substack{p \leqslant x \\ p \nmid q}} \frac{f(p)}{p}\right)
$$

in the range $q<y^{1-\beta}, x^{\alpha} \leqslant y \leqslant x$, where the implicit constant depends on $A$, $B, \alpha, \beta$. Shiu's result in $[12$ is actually stated in a slightly different way, which is however easily seen to be equivalent to the above. This was the first bound of this generality on sums of multiplicative functions on large subsequences of the integers, that is on arithmetic progressions in this case, and it proved to be very useful for different applications.

Nair [9] generalized Shiu's work to sums of the type $\sum_{n \leqslant x} f(|Q(n)|)$ with $f \in \mathcal{M}$ and $Q \in \mathbb{Z}[X]$. Nair and Tenenbaum [10] further generalized Nair's result to functions of several variables satisfying a property weaker than submultiplicativity. We quote their main result here. For fixed constants $k \geqslant 1, A \geqslant 1, B \geqslant 1, \varepsilon>0$, let $\mathcal{M}_{k}(A, B, \varepsilon)$ be the class of non-negative functions $F$ of $k$ variables such that

$$
F\left(a_{1} b_{1}, \ldots, a_{k} b_{k}\right) \leqslant \min \left(A^{\Omega\left(a_{1} \cdots a_{k}\right)}, B\left(a_{1} \cdots a_{k}\right)^{\varepsilon}\right) F\left(b_{1}, \ldots, b_{k}\right)
$$

for all $a_{i}, b_{j}$ such that $\left(a_{1} \cdots a_{k}, b_{1} \cdots b_{k}\right)=1$.

Theorem 1 (Nair and Tenenbaum). Let $k \geqslant 1$. Let $Q_{1}, \ldots, Q_{k} \in \mathbb{Z}[X]$ be $k$ pairwise coprime and irreducible polynomials. Let $Q=Q_{1} \cdots Q_{k}$ and denote by $g$ its degree and $D$ its discriminant. Let $\rho_{Q_{j}}(n)($ resp. $\rho(n))$ denote the number of zeroes of $Q_{j}$ (resp. $Q$ ) modulo $n$ for $1 \leqslant j \leqslant k$. Assume $Q$ has no fixed prime divisor. Let $0<\alpha<1,0<\delta<1, A \geqslant 1$ and $B \geqslant 1$. Let $\varepsilon \leqslant \frac{\alpha \delta}{12 g^{2}}$ and 
$F \in \mathcal{M}_{k}(A, B, \varepsilon)$. We have, uniformly in $x \geqslant c_{0}\|Q\|^{\delta}$ and $x^{\alpha}<y \leqslant x$,

$$
\begin{aligned}
& \sum_{x<n \leqslant x+y} F\left(\left|Q_{1}(n)\right|, \ldots,\left|Q_{k}(n)\right|\right) \\
& \ll y \prod_{p \leqslant x}\left(1-\frac{\rho(p)}{p}\right) \sum_{n_{1} \cdots n_{k} \leqslant x} F\left(n_{1}, \ldots, n_{k}\right) \frac{\rho_{Q_{1}}\left(n_{1}\right) \cdots \rho_{Q_{k}}\left(n_{k}\right)}{n_{1} \cdots n_{k}},
\end{aligned}
$$

where $c_{0}$ depends at most on $g, \alpha, \delta, A, B$ and the implicit constant depends at most on $g, D, \alpha, \delta, A, B$.

Actually, Nair and Tenenbaum do not require the polynomials $Q_{j}$ to be irreducible and pairwise coprime : this assumption is made here merely to simplify the statement of their result. Note that the implicit constant in (1.1) is allowed to depend on $D$. As a consequence of its generality, Nair and Tenenbaum's theorem can be extended to sums over integers $n$ in arithmetic progressions and to sums over primes $p$, as shown in 10 .

Daniel [3] obtained bounds of the type of (1.1) with uniformity in the discriminant $D$. In this article we are interested in obtaining such bounds and we improve on Daniel's results in several aspects, as we shall see later.

We first explain the motivation for our work. The need for bounds of type (1.1) uniform with respect to the discriminant of $Q$ has emerged in the context of several number-theoretic problems. One of these is the recent proof [6] of Quantum Unique Ergodicity by Soundararajan and Holowinsky, which combines different approaches by its two authors. Holowinsky's approach [5] relies on estimates for shifted convolution sums $\sum_{n \leqslant x} \lambda_{f}(n) \overline{\lambda_{f}(n+\ell)}$, where $\lambda_{f}$ are the renormalized Hecke eigenvalues of a Hecke eigencuspform $f$. These sums are averaged over $|\ell| \leqslant x$ in the course of Holowinsky's computations, therefore it is crucial to obtain an estimate uniform in $\operatorname{Disc}(X(X+\ell))=\ell^{2}$. The bound used by Holowinsky in [5] is the following, where we let $\tau_{m}$ denote the $m$-th divisor function and $\tau=\tau_{2}$.

Theorem 2 (Holowinsky). Let $\lambda_{1}$ and $\lambda_{2}$ be multiplicative functions such that the bound $\left|\lambda_{i}(n)\right| \leqslant \tau_{m}(n)$ holds for some $m$. Let $0<\varepsilon<1$, then for $x \geqslant c_{0}$ and uniformly in $1 \leqslant|\ell| \leqslant x$,

$$
\sum_{n \leqslant x}\left|\lambda_{1}(n) \lambda_{2}(n+\ell)\right| \ll \tau(|\ell|) \frac{x}{(\log x)^{2-\varepsilon}} \prod_{p \leqslant x}\left(1+\frac{\left|\lambda_{1}(p)\right|}{p}\right)\left(1+\frac{\left|\lambda_{2}(p)\right|}{p}\right),
$$

where $c_{0}$ and the implicit constant depend on $\varepsilon$ and $m$ at most.

Holowinsky's proof of the above result is based on the Large Sieve. Our results presented in this paper provide an independant proof of this theorem, together with a few refinements : $\tau(|\ell|)$ is replaced by a function $\Delta(\ell)$ with mean value 1 and the exponent $\varepsilon$ is removed. Another problem to feature discriminant-uniform bounds is the divisor problem for binary forms of degree 4 studied by Browning and de la Bretèche in 11. Their argument relies, among other things, on finding estimates [2] for the sums

$$
\sum_{n_{1} \leqslant X_{1}} \sum_{n_{2} \leqslant X_{2}} f\left(F\left(n_{1}, n_{2}\right)\right)
$$

where $f \in \mathcal{M}$ and $F$ is a binary form with non-zero discriminant. Their idea is to first study the inner sum with $n_{1}$ fixed so that $F\left(n_{1}, n_{2}\right)$ is a polynomial in $n_{2}$. For this sum they use an analogue of (1.1) (in the case $k=1$ ) with uniformity in the 
discriminant. Here again the uniformity is essential to average over $n_{1}$. Our results also apply in this case.

As stated above, the aim of this paper is to obtain discriminant-uniform bounds in the setting of Nair and Tenenbaum [10. We now introduce our main result. We restrict to the case of irreducible pairwise coprime polynomials $Q_{i}$ and multiplicative $F$ to simplify the exposition.

Theorem 3. Under the assumptions of Theorem 1, and assuming further that $F$ is multiplicative and that $\varepsilon \leqslant \frac{\alpha}{50 g(g+1 / \delta)}$, we have, uniformly in $x \geqslant c_{0}\|Q\|^{\delta}$ and $x^{\alpha}<y \leqslant x$,

$$
\begin{array}{rl}
\sum_{x<n \leqslant x+y} & F\left(\left|Q_{1}(n)\right|, \ldots,\left|Q_{k}(n)\right|\right) \\
& \ll \Delta_{D} y \prod_{p \leqslant x}\left(1-\frac{\rho(p)}{p}\right) \sum_{\substack{n_{1} \cdots n_{k} \leqslant x \\
\left(n_{1} \cdots n_{k}, D\right)=1}} F\left(n_{1}, \ldots, n_{k}\right) \frac{\rho_{Q_{1}}\left(n_{1}\right) \cdots \rho_{Q_{k}}\left(n_{k}\right)}{n_{1} \cdots n_{k}}
\end{array}
$$

where

$$
\Delta_{D}=\prod_{p \mid D}\left(1+\sum_{\substack{\nu_{j} \leqslant \operatorname{deg}\left(Q_{h}\right) \\(1 \leqslant j \leqslant k)}} F\left(p^{\nu_{1}}, \ldots, p^{\nu_{k}}\right) \frac{\#\left\{n \bmod p^{\max _{j}\left(\nu_{j}\right)+1}: p^{\nu_{j}} \| Q_{j}(n) \forall j\right\}}{p^{\max _{j}\left(\nu_{j}\right)+1}}\right) .
$$

The implicit constant and $c_{0}$ depend at most on $g, \alpha, \delta, A, B$.

Daniel [3] obtains a bound analogous to (1.2), with a method of proof different from us. However instead of $\Delta_{D}$, Daniel uses the weaker term $\tilde{\Delta}_{D}$ defined as $\Delta_{D}$ in (1.3) but where the conditions $p^{\nu_{j}} \| Q(n)$ are replaced by $p^{\nu_{j}} \mid Q(n)(1 \leqslant j \leqslant k)$. In the case $k=1$ we have

$$
\Delta_{D}=\prod_{p \mid D}\left(1+\sum_{\nu \leqslant g} F\left(p^{\nu}\right)\left(\frac{\rho\left(p^{\nu}\right)}{p^{\nu}}-\frac{\rho\left(p^{\nu+1}\right)}{p^{\nu+1}}\right)\right) \leqslant \tilde{\Delta}_{D}=\prod_{p \mid D}\left(1+\sum_{\nu \leqslant g} F\left(p^{\nu}\right) \frac{\rho\left(p^{\nu}\right)}{p^{\nu}}\right)
$$

which shows that the term $\Delta_{D}$ has the advantage of taking into account certain cancellations between values of the $\rho$ function. With this improved term $\Delta_{D}$, we are then able to show that the bound (1.2) is best possible in the sense that for all polynomials $Q_{i}$ and all constants $\alpha, \delta, A, B, \varepsilon$, it is attained for a large family of functions $F \in \mathcal{M}(A, B, \varepsilon)$. Our results are perhaps easier to apprehend in the setting of Shiu, in which they take the following form.

Theorem 4. Let $f \in \mathcal{M}$ and $Q \in \mathbb{Z}[X]$. Assume $Q$ is irreducible and denote by $g$ its degree and $D$ its discriminant. Let $0<\alpha<1$ and $0<\delta<1$. We have, uniformly in $x \geqslant c_{0}\|Q\|^{\delta}$ and $x^{\alpha}<y \leqslant x$,

$$
\sum_{x<n \leqslant x+y} f(|Q(n)|) \ll \Delta_{D} y \prod_{g<p \leqslant x}\left(1-\frac{\rho(p)}{p}\right) \exp \left(\sum_{\substack{p \leqslant x \\ p \nmid D}} \frac{f(p)}{p}\right)
$$

where the implicit constant and $c_{0}$ depend on $\alpha, \delta, A, B$ at most, and where $\Delta_{D}$ is defined by (1.4) (with $F=f$ ). 
In our article we use the method of proof of Nair and Tenenbaum in [10. To address the issue of preserving the uniformity in the discriminant, we employ the following bound by Stewart [13. For all primes $p$, we have

$$
\rho\left(p^{\nu}\right) \leqslant g p^{\left[\nu-\frac{\nu}{g}\right]} \quad(\nu \geqslant 1) .
$$

This allows us to bound the key quantity $\frac{\rho\left(p^{\nu}\right)}{p^{\nu}}$ by a negative power of $p^{\nu}$, whereas classical bounds by Nagell would only allows us to bound this quantity by a positive power of $p^{\nu}$ for $p \mid D$ and large $D$. Note that this idea was already present in the work of Daniel 3 .

The article is organized as follows. Section 2 is devoted to introducing the necessary notations. In Section 3 we state all of our results and we derive the theorems exposed in the introduction from them. In Section 4 we prove some technical lemmas that are of constant use in our argument, and in Sections 5, 6, 7 we prove our results.

Acknowledgements. I am very grateful to Régis de la Bretèche for suggesting this problem to me in the first place and for his guidance throughout the making of this paper. I would also like to thank Tim Browning and Gérald Tenenbaum for helpful suggestions. The research and writing of this work was carried during an internship of the author at the Université Paris 7 Denis Diderot whose hospitality is gratefully acknowledged.

\section{Notations AND DEFINitions}

We follow the notations of Nair and Tenenbaum in [10].

On integers. We let $P^{+}(n), P^{-}(n)$ respectively denote the greatest and the least prime factor of an integer $n$, with the convention that $P^{+}(1)=1$ and $P^{-}(1)=\infty$. We also let $[n]$ denote the greatest integer less than or equal to $n$.

We denote by $\Omega(n), \omega(n)$ the number of prime factors of $n$, counted respectively with or without multiplicity. We write $\varphi(n)$ for Euler's function and $\kappa(n)$ for the squarefree kernel of $n$, that is $\kappa(n)=\prod_{p \mid n} p$.

For $n, m \in \mathbb{N}$ we let $n \mid m^{\infty}$ indicate that all prime factors of $n$ divide $m$. The notation $a|| b$ means that $a \mid b$ and $\left(a, \frac{b}{a}\right)=1$.

On polynomials. For any $P \in \mathbb{Z}[X]$ we define $\|P\|$ as the sum of the coefficients of $P$ taken in absolute value, and we say that $p$ is a fixed prime divisor of $P$ when $p \mid Q(n)$ for all $n \in \mathbb{N}$.

For polynomials $Q_{1}, \ldots, Q_{k} \in \mathbb{Z}[X]$ we define $Q:=\prod_{j=1}^{k} Q_{j}$. We denote by $g$ the degree of $Q, r$ its number of irreducible factors and $D$ its discriminant. We assume that $Q$ is primitive, that is that the greatest common divisor of its coefficients is 1 .

We write the decomposition of these polynomials in irreducible factors as

$$
\begin{aligned}
Q & =R_{1}^{\gamma_{1}} \cdots R_{r}^{\gamma_{r}}, \\
Q_{j} & =R_{1}^{\gamma_{j 1}} \cdots R_{r}^{\gamma_{j r}}
\end{aligned}
$$

for $1 \leqslant j \leqslant k$. We define $Q^{*}:=R_{1} \cdots R_{r}$ and denote by $g^{*}$ its degree. We will mainly work with the polynomial $Q^{*}$ as it has the important property of having a non-zero discriminant, which we denote by $D^{*}$. For any polynomial $P \in \mathbb{Z}[X]$, we let $\rho_{P}(n)$ denote the number of zeroes of $P$ modulo $n$. We let

$$
\rho:=\rho_{Q}, \quad \rho^{*}:=\rho_{Q^{*}} .
$$


We next recall some well-known bounds (see e.g. [8]) on $\rho$ and $\rho^{*}$. For all primes $p$ we have

$$
\begin{aligned}
\rho(p) & \leqslant g, & & \\
\rho^{*}\left(p^{\nu}\right) & \leqslant g^{*} p^{\nu-1} & & (\nu \geqslant 1), \\
\rho^{*}\left(p^{\nu}\right) & =\rho^{*}(p) \leqslant g^{*} & & \left(p \nmid D^{*}, \nu \geqslant 1\right) .
\end{aligned}
$$

In our article we use in an essential way the following bounds by Stewart [13]. For all primes $p$, we have

$$
\begin{aligned}
\rho^{*}\left(p^{\nu}\right) & \leqslant g^{*} p^{\left[\nu-\frac{\nu}{g^{*}}\right]} \leqslant g p^{\left[\nu-\frac{\nu}{g}\right]} & & (\nu \geqslant 1) \\
\rho_{R_{h}}\left(p^{\nu}\right) & \leqslant \mu_{h} p^{\left[\nu-\frac{\nu}{\mu_{h}}\right]} & & (\nu \geqslant 1,1 \leqslant h \leqslant r)
\end{aligned}
$$

where $\mu_{h}=\operatorname{deg}\left(R_{h}\right)$. Finally we let

$$
\hat{\rho}_{\mathbf{R}}\left(n_{1}, \ldots, n_{r}\right)=\#\left\{n \bmod \left[n_{1} \kappa\left(n_{1}\right), \ldots, n_{r} \kappa\left(n_{r}\right)\right]: n_{h} \| R_{h}(n) \text { for } 1 \leqslant h \leqslant r\right\} .
$$

It is a multiplicative function. We record here an useful bound on $\hat{\rho}_{\mathbf{R}}$.

$$
\frac{\hat{\rho}_{\mathbf{R}}\left(n_{1}, \ldots, n_{r}\right)}{\left[n_{1} \kappa\left(n_{1}\right), \ldots, n_{r} \kappa\left(n_{r}\right)\right]} \leqslant \frac{\rho^{*}\left(n_{1} \cdots n_{r}\right)}{n_{1} \cdots n_{r}} .
$$

To see (2.9), note that

$$
\begin{aligned}
\frac{\hat{\rho}_{\mathbf{R}}\left(n_{1}, \ldots, n_{r}\right)}{\left[n_{1} \kappa\left(n_{1}\right), \ldots, n_{r} \kappa\left(n_{r}\right)\right]} & \leqslant \frac{\#\left\{n \bmod \left[n_{1}, \ldots, n_{r}\right]: n_{h} \mid R_{h}(n)(1 \leqslant h \leqslant r)\right\}}{\left[n_{1}, \ldots, n_{r}\right]} \\
& =\frac{\#\left\{n \bmod n_{1} \cdots n_{r}: n_{h} \mid R_{h}(n)(1 \leqslant h \leqslant r)\right\}}{n_{1} \cdots n_{r}} \\
& \leqslant \frac{\#\left\{n \bmod n_{1} \cdots n_{r}: n_{1} \cdots n_{r} \mid Q^{*}(n)\right\}}{n_{1} \cdots n_{r}} .
\end{aligned}
$$

On arithmetic functions. Let $H$ be a function of $s$ integer variables. We say that $H$ is submultiplicative (resp. multiplicative) if

$$
H\left(a_{1} b_{1}, \ldots, a_{s} b_{s}\right) \leqslant H\left(a_{1}, \ldots, a_{s}\right) H\left(b_{1}, \ldots, b_{s}\right)
$$

(resp. with equality in the above) for all $a_{i}, b_{j}$ such that $\left(a_{1} \cdots a_{s}, b_{1} \cdots b_{s}\right)=1$. We also define, for $1 \leqslant j \leqslant s$,

$$
H^{(j)}(n)=H(1, \ldots, n, \ldots, 1)
$$

where $n$ is at the $j$-th place.

We let $\mathcal{M}_{k}(A, B, \varepsilon)$ be the class of non-negative functions $F$ of $k$ integer variables satisfying

$$
F\left(a_{1} b_{1}, \ldots, a_{k} b_{k}\right) \leqslant \min \left(A^{\Omega\left(a_{1} \cdots a_{k}\right)}, B\left(a_{1} \cdots a_{k}\right)^{\varepsilon}\right) F\left(b_{1}, \ldots, b_{k}\right)
$$

for all $a_{i}, b_{j}$ such that $\left(a_{1} \cdots a_{k}, b_{1} \cdots b_{k}\right)=1$. Nair and Tenenbaum [10] actually consider functions $F$ satisfying the above property for all $a_{i}, b_{j}$ such that $\left(a_{i}, b_{i}\right)=1$, although the proof of their theorem requires this property only for integers $a_{i}, b_{j}$ such that $\left(a_{1} \cdots a_{k}, b_{1} \cdots b_{k}\right)=1$. We thus took the liberty of using the same notation as in 10 to denote our slightly larger class of functions. We remark here that $F$ is zero if $F(1, \ldots, 1)=0$. 
For a function $F$ of $k$ variables such that $F(1, \ldots, 1) \neq 0$, we define an associated minimal function

$$
G\left(a_{1}, \ldots, a_{k}\right)=\max _{\substack{b_{1}, \ldots, b_{k} \geqslant 1 \\\left(a_{1} \cdots a_{k}, b_{1} \cdots b_{k}\right)=1 \\ F\left(b_{1}, \ldots, b_{k}\right) \neq 0}} \frac{F\left(a_{1} b_{1}, \ldots, a_{k} b_{k}\right)}{F\left(b_{1}, \ldots, b_{k}\right)} .
$$

Note that $G=F$ when $F$ is multiplicative. When $F \in \mathcal{M}_{k}(A, B, \varepsilon)$, it is easily checked that $G$ is submultiplicative and

$$
\begin{aligned}
& G\left(n_{1}, \ldots, n_{k}\right) \leqslant \min \left(A^{\Omega\left(n_{1} \cdots n_{k}\right)}, B\left(n_{1} \cdots n_{k}\right)^{\varepsilon}\right), \\
& G\left(n_{1}, \ldots, n_{k}\right) \leqslant \prod_{p^{\nu} \| n_{1} \cdots n_{k}} \min \left(A^{\nu}, B p^{\varepsilon \nu}\right) .
\end{aligned}
$$

Special notation. We let $F$ be a function of $k$ variables such that $F(1, \ldots, 1) \neq 0$. Decomposing polynomials $Q_{j}(1 \leqslant j \leqslant k)$ as in (2.2), we remark that

$$
F\left(\left|Q_{1}(n)\right|, \ldots,\left|Q_{k}(n)\right|\right)=\tilde{F}\left(\left|R_{1}(n)\right|, \ldots,\left|R_{r}(n)\right|\right) \quad(n \geqslant 1)
$$

where $\tilde{F}$ is defined by

$$
\tilde{F}\left(n_{1}, \ldots, n_{r}\right):=F\left(n_{1}^{\gamma_{11}} \cdots n_{r}^{\gamma_{1 r}}, \ldots, n_{1}^{\gamma_{k 1}} \cdots n_{r}^{\gamma_{k r}}\right) .
$$

If $G$ is the minimal function associated to $F$ by (2.11), then $\tilde{G}$ is the minimal function associated to $\tilde{F}$ in a similar fashion. Therefore

$$
\tilde{F}\left(a_{1} b_{1}, \ldots, a_{r} b_{r}\right) \leqslant \tilde{G}\left(a_{1}, \ldots, a_{r}\right) \tilde{F}\left(b_{1}, \ldots, b_{r}\right)
$$

for all $a_{i}, b_{j}$ such that $\left(a_{1} \cdots a_{r}, b_{1} \cdots b_{r}\right)=1$. When $F \in \mathcal{M}_{k}(A, B, \varepsilon)$ we obviously have $\tilde{F} \in \mathcal{M}_{r}\left(A^{g}, B, g \varepsilon\right)$ and therefore by (2.12) and (2.13) we have

$$
\begin{aligned}
& \tilde{G}\left(n_{1}, \ldots, n_{r}\right) \leqslant A^{g \Omega\left(n_{1} \cdots n_{r}\right)}, \\
& \tilde{G}\left(n_{1}, \ldots, n_{r}\right) \leqslant \prod_{p^{\nu} \| n_{1} \cdots n_{r}} \min \left(A^{g \nu}, B p^{g \varepsilon \nu}\right) .
\end{aligned}
$$

\section{RESUlts}

Our main theorem is the following.

Theorem 5. Let $k$ be a positive integer and let $Q_{j} \in \mathbb{Z}[X](1 \leqslant j \leqslant k)$. Let $Q=\prod_{j=1}^{k} Q_{j}$ and assume that $Q$ is primitive. Let (2.1) be the decomposition of $Q$ in irreducible factors and define $g, \rho, \hat{\rho}_{\mathbf{R}}$ as in the previous section. Let $0<\alpha<1$, $0<\delta<1, A \geqslant 1$ and $B \geqslant 1$. Let also $0<\varepsilon<\frac{\alpha}{50 g\left(g+\frac{1}{\delta}\right)}$ and $F \in \mathcal{M}_{k}(A, B, \varepsilon)$. Then we have, uniformly in $x \geqslant c_{0}\|Q\|^{\delta}$ and $x^{\alpha} \leqslant y \leqslant x$,

$$
\begin{aligned}
\sum_{x<n \leqslant x+y} F( & \left.\left|Q_{1}(n)\right|, \ldots,\left|Q_{k}(n)\right|\right) \\
& \ll y \prod_{g<p \leqslant x}\left(1-\frac{\rho(p)}{p}\right) \sum_{n_{1} \cdots n_{r} \leqslant x} \tilde{F}\left(n_{1}, \ldots, n_{r}\right) \frac{\hat{\rho}_{\mathbf{R}}\left(n_{1}, \ldots, n_{r}\right)}{\left[n_{1} \kappa\left(n_{1}\right), \ldots, n_{r} \kappa\left(n_{r}\right)\right]}
\end{aligned}
$$

where $c_{0}$ and the implicit constant depend at most on $g, \alpha, \delta, A, B$.

We also provide a bound in which the dependency on the discriminant $D^{*}$ is made explicit. 
Corollary 1. Under the assumptions of Theorem 5 ,

$$
\begin{aligned}
& \sum_{x<n \leqslant x+y} F\left(\left|Q_{1}(n)\right|, \ldots,\left|Q_{k}(n)\right|\right) \\
& \ll \Delta_{D^{*}} y \prod_{g<p \leqslant x}\left(1-\frac{\rho(p)}{p}\right) \sum_{\substack{n_{1} \cdots n_{r} \leqslant x \\
\left(n_{1} \cdots n_{r}, D^{*}\right)=1 \\
\left(n_{i}, n_{j}\right)=1(i \neq j)}} \tilde{F}\left(n_{1}, \ldots, n_{r}\right) \frac{\rho_{R_{1}}\left(n_{1}\right) \cdots \rho_{R_{r}}\left(n_{r}\right)}{n_{1} \cdots n_{r}}
\end{aligned}
$$

where

$$
\Delta_{D^{*}}=\prod_{p \mid D^{*}}\left(1+\sum_{\substack{\nu_{h} \leqslant \operatorname{deg}\left(R_{h}\right) \\(1 \leqslant h \leqslant r)}} \tilde{G}\left(p^{\nu_{1}}, \cdots, p^{\nu_{r}}\right) \frac{\hat{\rho}_{\mathbf{R}}\left(p^{\nu_{1}}, \ldots, p^{\nu_{r}}\right)}{p^{\max \left(\nu_{h}\right)+1}}\right) .
$$

The dependencies of the various constants are as described in Theorem 5 .

Remark. Using (2.9) and the trivial bound (2.4) on $\rho^{*}$, we see that

$$
1 \leqslant \Delta_{D^{*}} \leqslant \prod_{p \mid D^{*}}\left(1+\frac{1}{p}\right)^{C}
$$

with $C=g \cdot \max _{p} \sum_{\nu_{h} \leqslant \operatorname{deg}\left(R_{h}\right)(1 \leqslant h \leqslant r)} \tilde{G}\left(p^{\nu_{1}}, \ldots, p^{\nu_{r}}\right)$. Therefore $\Delta_{D^{*}}$ has mean value one when averaged over $D^{*}$.

Corollary 2. Under the assumptions of Theorem 5 ,

$$
\begin{aligned}
\sum_{x<n \leqslant x+y} F\left(\left|Q_{1}(n)\right|, \ldots,\left|Q_{k}(n)\right|\right) & \\
& \ll \Delta_{D^{*}} y \prod_{g<p \leqslant x}\left(1-\frac{\rho(p)}{p}\right) \prod_{\substack{p \leqslant x \\
p \nmid D^{*}}} \prod_{h=1}^{r}\left(1+\tilde{G}^{(h)}(p) \frac{\rho_{R_{h}}(p)}{p}\right)
\end{aligned}
$$

where $\Delta_{D^{*}}$ is defined by (3.2). The dependencies of the various constants are as described in Theorem 5 .

This corollary sheds some light on the difference of behavior between the part of the sum that depends on $D^{*}$ and the part that is independant of $D^{*}$. Indeed for primes $p \nmid D^{*}$, only the values $\tilde{G}(1, \ldots, p, \ldots, 1)$, where $p$ is at the $h$-th place $(1 \leqslant h \leqslant r)$, are involved in the bound, whereas for primes $p \mid D^{*}$ we have to take into account the values $\tilde{G}\left(p^{\nu_{1}}, \ldots, p^{\nu_{r}}\right)$ for $\nu_{h} \leqslant \operatorname{deg}\left(R_{h}\right)(1 \leqslant h \leqslant r)$. As will be shown in the proof, this is due to the fact that $\rho^{*}\left(p^{\nu}\right)$ is bounded when $p \nmid D^{*}$, whereas it can be very large when $p \mid D^{*}$. It can indeed be as large as the right-hand side of (2.6) as shown by Stewart [13.

Our second theorem gives an order of magnitude instead of an upper bound.

Theorem 6. Under the assumptions of Theorem 5, and assuming further that $Q$ has no fixed prime divisor, $F$ is multiplicative and

$$
F\left(n_{1}, \ldots, n_{k}\right) \gg \eta^{\Omega\left(n_{1} \ldots n_{k}\right)} \quad\left(n_{1}, \ldots, n_{k} \geqslant 1\right)
$$


for some $\eta \in] 0,1[$, we have

$$
\begin{array}{rl}
\sum_{x<n \leqslant x+y} & F\left(\left|Q_{1}(n)\right|, \ldots,\left|Q_{k}(n)\right|\right) \\
(3.4) \quad & \asymp y \prod_{g<p \leqslant x}\left(1-\frac{\rho(p)}{p}\right) \sum_{n_{1} \cdots n_{r} \leqslant x} \tilde{F}\left(n_{1}, \ldots, n_{r}\right) \frac{\hat{\rho}_{\mathbf{R}}\left(n_{1}, \ldots, n_{r}\right)}{\left[n_{1} \kappa\left(n_{1}\right), \ldots, n_{r} \kappa\left(n_{r}\right)\right]} \\
(3.5) \quad & \asymp \Delta_{D^{*}} y \prod_{g<p \leqslant x}\left(1-\frac{\rho(p)}{p}\right) \prod_{\substack{p \leqslant x \\
p \nmid D^{*}}} \prod_{h=1}^{r}\left(1+\tilde{F}^{(h)}(p) \frac{\rho_{R_{h}}(p)}{p}\right)
\end{array}
$$

where $\Delta_{D^{*}}$ is defined by (3.2) and the implied constant depends at most on $g, \alpha$, $\delta, A, B, \eta$.

Thus when $F$ is multiplicative and doesn't take too small values in the sense above, the bound we obtain in Theorem 5 is sharp. The $D^{*}$-dependency of the sum is accurately given by $\Delta_{D^{*}}$ in this case.

Eventually we provide the following result analogous to Theorem 3 of Nair of Tenenbaum [10], to illustrate how the generality of Theorem [5 can be used.

Theorem 7. Under the assumptions of Theorem [5, and provided that $Q(0) \neq 0$, we have

$$
\begin{aligned}
& \sum_{x<p \leqslant x+y} F\left(\left|Q_{1}(p)\right|, \ldots,\left|Q_{k}(p)\right|\right) \ll \frac{Q(0)}{\varphi(Q(0))} \Delta_{D^{*}} \frac{y}{\log x} \prod_{g<p \leqslant x}\left(1-\frac{\rho(p)}{p}\right) \\
& \times \sum_{n_{1} \cdots n_{r} \leqslant x} \tilde{F}\left(n_{1}, \ldots, n_{r}\right) \frac{\hat{\rho}_{\mathbf{R}}\left(n_{1}, \ldots, n_{r}\right)}{\left[n_{1} \kappa\left(n_{1}\right), \ldots, n_{r} \kappa\left(n_{r}\right)\right]} .
\end{aligned}
$$

where $\Delta_{D^{*}}$ is defined by (3.2). The dependencies of the various constants are as described in Theorem 5 .

We refer to [10, Proof of Theorem 3] for the proof of this Theorem as it is absolutely analogous in our setting.

It is easy to derive the theorems of the introduction from the previous results. Theorem 3 follows immediately from Corollary 1 upon observing that when the $Q_{i}$ are irreducible and $F$ is multiplicative we have $F=\tilde{F}=G=\tilde{G}, k=r$ and $Q_{i}=R_{i}$ for $1 \leqslant i \leqslant k$. Theorem 4 is similarly derived from Corollary 2 . We can also recover Theorem 2 of Holowinsky with the refinements mentioned in the introduction by applying Corollary 1 and its following remark with $Q_{1}=X, Q_{2}=X+\ell$ and $F\left(n_{1}, n_{2}\right)=\lambda_{1}\left(n_{1}\right) \lambda_{2}\left(n_{2}\right)$.

The rest of this article is dedicated to proving Theorems 5 , 6 and Corollaries 1 , 2 which share the same hypotheses (except for some additional assumptions for Theorem [6). We therefore place ourselves under the assumptions of Theorem 5 for the remaining sections. We also assume that $F$ is non-zero and further that $F(1, \ldots, 1)=1$, which is possible upto multipliying $F$ by a certain constant. All implicit constants throughout the article will depend at most on $g, \alpha, \delta, A, B, \varepsilon$ unless otherwise stated.

\section{TECHNiCAL LEMMAS}

The purpose of this section is to expose a few technical lemmas inspired by Lemma 1 and Lemma 2 by Nair and Tenenbaum in [10. 
We first have to introduce the functions these lemmas will apply to and their properties.

Lemma 1. Let $\sigma_{1}, \ldots, \sigma_{r}$ ber positive multiplicative functions satisfying $\sigma_{h}\left(p^{\nu}\right) \ll 1$ uniformly in primes $p$ and integers $\nu \geqslant 1(1 \leqslant h \leqslant r)$. Define

$$
\begin{aligned}
H\left(n_{1}, \ldots, n_{r}\right) & :=\tilde{F}\left(n_{1}, \ldots, n_{r}\right) \frac{\hat{\rho}_{\mathbf{R}}\left(n_{1}, \ldots, n_{r}\right)}{\left[n_{1} \kappa\left(n_{1}\right), \ldots, n_{r} \kappa\left(n_{r}\right)\right]} \sigma_{1}\left(n_{1}\right) \cdots \sigma_{r}\left(n_{r}\right), \\
T\left(n_{1}, \ldots, n_{r}\right) & :=\tilde{G}\left(n_{1}, \ldots, n_{r}\right) \frac{\hat{\rho}_{\mathbf{R}}\left(n_{1}, \ldots, n_{r}\right)}{\left[n_{1} \kappa\left(n_{1}\right), \ldots, n_{r} \kappa\left(n_{r}\right)\right]} \sigma_{1}\left(n_{1}\right) \cdots \sigma_{r}\left(n_{r}\right) .
\end{aligned}
$$

We then have

$$
H\left(a_{1} b_{1}, \ldots, a_{r} b_{r}\right) \leqslant T\left(b_{1}, \ldots, b_{r}\right) H\left(a_{1}, \ldots, a_{r}\right)
$$

for all integers $a_{i}, b_{j}$ such that $\left(a_{1} \cdots a_{r}, b_{1} \cdots b_{r}\right)=1$. We also hav 1

$$
\begin{aligned}
\sum_{\nu_{1}, \ldots, \nu_{r}}^{\prime} T\left(p^{\nu_{1}}, \ldots, p^{\nu_{r}}\right) & \ll \frac{1}{p}, \\
\sum_{\nu_{1}+\cdots+\nu_{r}>2 g} T\left(p^{\nu_{1}}, \ldots, p^{\nu_{r}}\right) \cdot p^{\frac{1}{4 g}\left(\nu_{1}+\cdots+\nu_{r}\right)} & \ll \frac{1}{p^{1+1 / 4}} .
\end{aligned}
$$

Proof. The inequality (4.2) follows immediately from (2.15) and the multiplicativity of $\hat{\rho}_{\mathbf{R}}$.

To obtain the two next bounds on $T$, we apply (2.9), (2.17) and the bounds $\sigma_{h}\left(p^{\nu}\right) \ll 1(1 \leqslant h \leqslant r)$ to obtain

$$
T\left(p^{\nu_{1}}, \ldots, p^{\nu_{r}}\right) \ll \min \left(A^{g \nu}, B p^{g \varepsilon \nu}\right) \frac{\rho^{*}\left(p^{\nu}\right)}{p^{\nu}}
$$

with $\nu=\nu_{1}+\cdots+\nu_{r}$. Using Stewart's bound (2.6) on $\rho^{*}$, we obtain

$$
\sum_{\nu_{1}+\cdots+\nu_{r}>2 g} T\left(p^{\nu_{1}}, \ldots, p^{\nu_{r}}\right) \cdot p^{\frac{1}{4 g}\left(\nu_{1}+\cdots+\nu_{r}\right)} \ll \sum_{\nu>2 g} p^{\left(g \varepsilon+\frac{1}{4 g}-\frac{1}{g}\right) \nu} \nu^{r} \ll \frac{1}{p^{1+c}}
$$

with $c=\frac{23}{50} \geqslant \frac{1}{4}$. This proves (4.4), and to prove (4.3) we can now restrict ourselves to the (finite) sum over the $\nu_{i}$ such that $\nu_{1}+\cdots+\nu_{r} \leqslant 2 g$. For these $\nu_{i}$ we have $T\left(p^{\nu_{1}}, \ldots, p^{\nu_{r}}\right) \ll \frac{1}{p}$ by (4.5) and (2.4), which concludes the proof.

Lemma 2. Let $H$ be as in Lemma 1 and let $\theta_{1}, \ldots, \theta_{r}$ be $r$ positive multiplicative functions satisfying $\theta_{h}\left(p^{\nu}\right)=1+O\left(\frac{1}{p}\right)$ uniformly in primes $p$ and integers $\nu \geqslant 1$ for all $1 \leqslant h \leqslant r$. We have, uniformly in $z>0$,

$$
\sum_{n_{1} \cdots n_{r} \leqslant z} H\left(n_{1}, \ldots, n_{r}\right) \theta_{1}\left(n_{1}\right) \cdots \theta_{r}\left(n_{r}\right) \ll \sum_{n_{1} \cdots n_{r} \leqslant z} H\left(n_{1}, \ldots, n_{r}\right) .
$$

Proof. For $1 \leqslant h \leqslant r$ and integers $n_{h}$, write

$$
\theta_{h}\left(n_{h}\right)=\sum_{d_{h} \mid n_{h}} \lambda_{h}\left(d_{h}\right)
$$

\footnotetext{
${ }^{1}$ Here and in the sequel the prime next to the sum indicates that the sum is over variables which are not all zero.
} 
KEVIN HENRIOT

We have $\lambda_{h}\left(p^{\nu}\right)=\theta_{h}\left(p^{\nu}\right)-\theta_{h}\left(p^{\nu-1}\right) \ll \frac{1}{p}$ for $\nu \geqslant 1$. For any integers $d_{h}, n_{h}$ such that $d_{h} \mid n_{h}$, we can write $n_{h}$ uniquely as $n_{h}=d_{h} t_{h} a_{h}$ with $t_{h} \mid d_{h}^{\infty}$ and $\left(a_{h}, d_{h}\right)=1$. Using (4.2) and (4.6) we obtain

$$
\begin{array}{rl}
\sum_{n_{1} \cdots n_{r} \leqslant z} & H\left(n_{1}, \ldots, n_{r}\right) \theta_{1}\left(n_{1}\right) \cdots \theta_{r}\left(n_{r}\right) \\
& \leqslant \sum_{d_{1}, \ldots, d_{r}} \sum_{\substack{t_{1}, \ldots, t_{r} \\
t_{h} \mid d_{h}^{+}}} \lambda_{1}\left(d_{1}\right) \cdots \lambda_{r}\left(d_{r}\right) T\left(d_{1} t_{1}, \ldots, d_{r} t_{r}\right) \sum_{a_{1} \cdots a_{r} \leqslant z} H\left(a_{1}, \ldots, a_{r}\right) \\
& \leqslant \Delta_{1} \sum_{a_{1} \cdots a_{r} \leqslant z} H\left(a_{1}, \ldots, a_{r}\right)
\end{array}
$$

where

$$
\Delta_{1}=\prod_{p}\left(1+\sum_{s_{1}, \ldots, s_{r}}^{\prime} \lambda_{1}\left(p^{s_{1}}\right) \cdots \lambda_{r}\left(p^{s_{r}}\right) \sum_{\ell_{1}, \ldots, \ell_{r}} T\left(p^{s_{1}+\ell_{1}}, \ldots, p^{s_{r}+\ell_{r}}\right)\right) .
$$

Now by (4.3) and the bound $\lambda_{h}\left(p^{\nu}\right) \ll \frac{1}{p}$, we have

$$
\Delta_{1}=\prod_{p}\left(1+O\left(\frac{1}{p^{2}}\right)\right) \ll 1
$$

which concludes the proof.

Lemma 3. Let $H$ be as in Lemma 1. Then for $\chi>0, z \geqslant e^{4 g \chi}, \beta=\frac{\chi}{\log z}$,

$$
\sum_{P^{+}\left(n_{1} \cdots n_{r}\right) \leqslant z} H\left(n_{1}, \ldots, n_{r}\right)\left(n_{1} \cdots n_{r}\right)^{\beta} \ll_{\chi} \sum_{P^{+}\left(n_{1} \cdots n_{r}\right) \leqslant z} H\left(n_{1}, \ldots, n_{r}\right) .
$$

Proof. For any integer $n$ write $n^{\beta}=\sum_{d \mid n} \psi(d)$. For any integers $d, n$ such that $d \mid n$ we can write $n$ uniquely as $n=d t a, t \mid d^{\infty},(a, d)=1$. Applying (4.2), we obtain

$$
\begin{aligned}
& \sum_{P^{+}\left(n_{1} \cdots n_{r}\right) \leqslant z} H\left(n_{1}, \ldots, n_{r}\right)\left(n_{1} \cdots n_{r}\right)^{\beta} \\
\leqslant & \sum_{P^{+}\left(d_{1} \cdots d_{r}\right) \leqslant z} \sum_{\substack{t_{1}, \ldots, t_{r} \\
t_{h}, d_{h}^{\prime}}} \psi\left(d_{1}\right) \cdots \psi\left(d_{r}\right) T\left(d_{1} t_{1}, \ldots, d_{r} t_{r}\right) \sum_{P^{+}\left(a_{1} \cdots a_{r}\right) \leqslant z} H\left(a_{1}, \ldots, a_{r}\right) \\
\leqslant & \Delta_{2} \sum_{P^{+}\left(a_{1} \cdots a_{r}\right) \leqslant z} H\left(a_{1}, \ldots, a_{r}\right)
\end{aligned}
$$

where

$$
\Delta_{2}=\prod_{p \leqslant z} \sum_{s_{1}, \ldots, s_{r}} \psi\left(p^{s_{1}}\right) \cdots \psi\left(p^{s_{r}}\right) \sum_{\substack{\ell_{1}, \ldots, \ell_{r} \geqslant 0 \\ \ell_{h}=0 \text { if } s_{h}=0}} T\left(p^{s_{1}+\ell_{1}}, \ldots, p^{s_{r}+\ell_{r}}\right) .
$$

We can rewrite this as

$$
\begin{aligned}
\Delta_{2} & =\prod_{p \leqslant z}\left(1+\sum_{\nu_{1}, \ldots, \nu_{r}}^{\prime} T\left(p^{\nu_{1}}, \ldots, p^{\nu_{r}}\right) \prod_{\substack{1 \leqslant h \leqslant r \\
\nu_{h} \neq 0}} \sum_{k=1}^{\nu_{h}} \psi\left(p^{k}\right)\right) \\
& =\prod_{p \leqslant z}\left(1+\sum_{\nu_{1}, \ldots, \nu_{r}}^{\prime} T\left(p^{\nu_{1}}, \ldots, p^{\nu_{r}}\right) \prod_{\substack{1 \leqslant h \leqslant r \\
\nu_{h} \neq 0}}\left(p^{\beta \nu_{h}}-1\right)\right) .
\end{aligned}
$$


We bound the inner product by distinguishing two cases. If $1 \leqslant \nu_{1}+\cdots+\nu_{r} \leqslant 2 g$ we have, for all $h, \beta \nu_{h} \log p \leqslant 2 \chi g \frac{\log p}{\log z} \ll_{\chi} 1$. Therefore for all $h, p^{\nu_{h} \beta}-1 \ll_{\chi} \frac{\log p}{\log z}$ which is also $\ll_{\chi} 1$. Since at least one $\nu_{h}$ is $\neq 0$ we have

$$
\prod_{\substack{1 \leqslant h \leqslant r \\ \nu_{h} \neq 0}}\left(p^{\beta \nu_{h}}-1\right) \ll_{\chi} \frac{\log p}{\log z}
$$

If $\nu_{1}+\cdots+\nu_{r}>2 g$ we use the trivial bound

$$
\prod_{\substack{1 \leqslant h \leqslant r \\ \nu_{h} \neq 0}}\left(p^{\beta \nu_{h}}-1\right) \leqslant p^{\beta\left(\nu_{1}+\cdots+\nu_{r}\right)} \leqslant p^{\frac{1}{4 g}\left(\nu_{1}+\cdots+\nu_{r}\right)} .
$$

Combining this with our bounds (4.3) and (4.4) on $T$ we arrive at

$$
\begin{aligned}
\Delta_{2} & =\prod_{p \leqslant z}\left(1+O_{\chi}\left(\frac{1}{\log z} \frac{\log p}{p}+\frac{1}{p^{1+1 / 4}}\right)\right) \\
& \leqslant \exp \left(O_{\chi}\left(\frac{1}{\log z} \sum_{p \leqslant z} \frac{\log p}{p}+\sum_{p \leqslant z} \frac{1}{p^{1+1 / 4}}\right)\right) \ll_{\chi} 1 .
\end{aligned}
$$

Lemma 4. Let $H$ be as in Lemma 1 and $K>0$. We have, uniformly in $z>0$,

$$
\sum_{P^{+}\left(n_{1} \cdots n_{r}\right) \leqslant z} H\left(n_{1}, \ldots, n_{r}\right) \leqslant K^{O(1)} \sum_{P^{+}\left(n_{1} \cdots n_{r}\right) \leqslant z^{1 / K}} H\left(n_{1}, \ldots, n_{r}\right) .
$$

Proof. For all $1 \leqslant h \leqslant r$ we write $n_{h}=a_{h} b_{h}$ where $P^{+}\left(a_{h}\right) \leqslant z^{\frac{1}{K}}$ and $P^{-}\left(b_{h}\right)>z^{\frac{1}{K}}$. Applying (4.2), we obtain

$$
\begin{aligned}
\sum_{P^{+}\left(n_{1} \cdots n_{r}\right) \leqslant z} H\left(n_{1}, \ldots, n_{r}\right) & \leqslant \sum_{\substack{P^{+}\left(b_{1} \cdots b_{r}\right) \leqslant z \\
P^{-}\left(b_{1} \cdots b_{r}\right)>z^{1 / K}}} T\left(b_{1}, \ldots, b_{r}\right) \sum_{P^{+}\left(a_{1} \cdots a_{r}\right) \leqslant z^{1 / K}} H\left(a_{1}, \ldots, a_{r}\right) \\
& \leqslant\left(\prod_{z^{1 / K}<p \leqslant z} \sum_{\nu_{1}, \ldots, \nu_{r}} T\left(p^{\nu_{1}}, \ldots, p^{\nu_{r}}\right)\right) \sum_{P^{+}\left(a_{1} \cdots a_{r}\right) \leqslant z^{1 / K}} H\left(a_{1}, \ldots, a_{r}\right) .
\end{aligned}
$$

To conclude we observe that by (4.3) the product above is

$$
\leqslant \prod_{z^{1 / K}<p \leqslant z}\left(1+\frac{1}{p}\right)^{O(1)} \leqslant K^{O(1)} .
$$

Lemma 5. Let $H$ be as in Lemma 1. We have, uniformly in $z>0$,

$$
\sum_{P^{+}\left(n_{1} \cdots n_{r}\right) \leqslant z} H\left(n_{1}, \ldots, n_{r}\right) \asymp \sum_{n_{1} \cdots n_{r} \leqslant z} H\left(n_{1}, \ldots, n_{r}\right) .
$$


Proof. The lower bound is obvious. To prove the upper bound, we introduce a constant $K>0$ whose value will be determined later. By Lemma 4 there exists $L>0$ depending on the usual parameters such that

$$
\begin{aligned}
\sum_{P^{+}\left(n_{1} \cdots n_{r}\right) \leqslant z} H\left(n_{1}, \ldots, n_{r}\right) & \leqslant K^{L} \sum_{P^{+}\left(n_{1} \cdots n_{r}\right) \leqslant z^{1 / K}} H\left(n_{1}, \ldots, n_{r}\right) \\
& \leqslant U+K^{L} \sum_{n_{1} \cdots n_{r} \leqslant z} H\left(n_{1}, \ldots, n_{r}\right)
\end{aligned}
$$

where

$$
U=K^{L} \sum_{\substack{\left.n_{1} \cdots n_{r}>z \\ P_{1} \cdots n_{r}\right) \leqslant z^{1 / K}}} H\left(n_{1}, \ldots, n_{r}\right) .
$$

We let $\beta_{K}=\frac{1}{\log \left(z^{1 / K}\right)}=\frac{K}{\log z}$. For $z \geqslant e^{4 g K}$, we have

$$
\begin{aligned}
U & \leqslant K^{L} z^{-\beta_{K}} \sum_{P^{+}\left(n_{1} \cdots n_{r}\right) \leqslant z^{1 / K}} H\left(n_{1}, \ldots, n_{r}\right)\left(n_{1} \cdots n_{r}\right)^{\beta_{K}} \\
& \ll K^{L} e^{-K} \sum_{P^{+}\left(n_{1} \cdots n_{r}\right) \leqslant z^{1 / K}} H\left(n_{1}, \ldots, n_{r}\right)
\end{aligned}
$$

where we have used Lemma 3 with $\chi=1$ in the second step. For a good choice of $K$ (depending on the usual parameters) we can thus impose

$$
U \leqslant \frac{1}{2} \sum_{P^{+}\left(n_{1} \cdots n_{r}\right) \leqslant z} H\left(n_{1}, \ldots, n_{r}\right) .
$$

Inserting this back into (4.8) yields the desired bound for $z$ large enough. When $z$ is bounded, so is the left-hand side of (4.7) by (4.2), (4.3) and (4.4). Since the right-hand side is superior to $H(1, \ldots, 1)=1$, (4.7) still holds in this case.

\section{Proof of Theorem 5}

In this section we prove Theorem [5, following closely the proof of Theorem 1 in [10] with occasional modifications to preserve the uniformity in the discriminant.

We define

$$
\varepsilon_{1}:=\frac{3}{25} \alpha, \quad \varepsilon_{2}:=\frac{\varepsilon_{1}}{3}, \quad \varepsilon_{3}:=\frac{\varepsilon_{1}}{6 g} .
$$

Before proceeding to the proof of Theorem 5 we establish the following sieve bound, which is essential to our argument.

Lemma 6. Let $\Xi$ be the set of fixed prime divisors of $Q$. Assume $z \leqslant x^{\varepsilon_{3}}$ and $a_{1} \cdots a_{r} \leqslant x^{\varepsilon_{1}}$. Then for $z$ large enough,

$$
\sum_{\substack{x<n \leqslant x+y \\ a_{h} \|, R_{h}(n)(1 \leqslant h \leqslant r) \\ p|Q(n) \Rightarrow p| a_{1} \cdots a_{r} \\ \text { or } p \in \Xi \text { or } p>z}} \asymp y \frac{\hat{\rho}_{\mathbf{R}}\left(a_{1}, \ldots, a_{r}\right)}{\left[a_{1} \kappa\left(a_{1}\right), \ldots, a_{r} \kappa\left(a_{r}\right)\right]} \prod_{\substack{g<p \leqslant z \\ p \nmid a_{1} \cdots a_{r}}}\left(1-\frac{\rho(p)}{p}\right) .
$$

Proof. We use Brun's sieve as exposed by Halberstam and Richert in [4, following their notations. We define a sequence

$$
\mathcal{A}:=\left\{Q(n): x<n \leqslant x+y \text { such that } a_{h} \| R_{h}(n)(1 \leqslant h \leqslant r)\right\}
$$


and a sifting set of primes

$$
\mathcal{B}:=\left\{p \notin \Xi \text { such that } p \nmid a_{1} \cdots a_{r}\right\} .
$$

With these definitions the left-hand side of (5.2) is nothing more than $S(\mathcal{A}, \mathcal{B}, z)$. We have

$$
X=y \frac{\hat{\rho}_{\mathbf{R}}\left(a_{1}, \ldots, a_{r}\right)}{\left[a_{1} \kappa\left(a_{1}\right), \ldots, a_{r} \kappa\left(a_{r}\right)\right]}
$$

and for $d$ squarefree with prime factors in $\mathcal{B}$,

$$
\begin{aligned}
\omega(d) & =\rho(d), \\
\left|R_{d}\right| & \leqslant \hat{\rho}_{\mathbf{R}}\left(a_{1}, \ldots, a_{r}\right) \rho(d) .
\end{aligned}
$$

We first check that $X \geqslant y /\left(a_{1} \cdots a_{r}\right)^{2} \geqslant x^{\alpha-2 \varepsilon_{1}} \geqslant x^{\frac{19}{25} \alpha}>1$. We also have $\omega(p) \leqslant g$ and

$$
0 \leqslant \frac{\omega(p)}{p} \leqslant 1-\frac{1}{g+1}
$$

for $p \in \mathcal{B}$, so that $\left(\Omega_{0}\right)$ holds with $A_{0}=g$ and $\left(\Omega_{1}\right)$ holds with $A_{1}=g+1$. Lemma 2.2 p.52 of 4 then implies that $\left(\Omega_{2}(\kappa)\right)$ holds with $\kappa=A_{0}=A_{2}=g$. The condition $(R)$ is also satisfied in its modified form

$$
\left|R_{d}\right| \leqslant L \omega(d)
$$

with $L=\hat{\rho}_{R}\left(a_{1}, \ldots, a_{r}\right) \leqslant\left(a_{1} \cdots a_{r}\right)^{2} \leqslant x^{2 \varepsilon_{1}}$. We can therefore apply Theorem 2.1 p.57 of 4 together with its Remark 2 , with the choice of parameters $b=1$ and $\lambda=\frac{1}{2 e}$. This yields, for $z$ large enough (with respect to the $A_{i}$ and $\kappa$, that is with respect to $g$ in our setting),

$$
S(\mathcal{A}, \mathcal{B}, z)=v X W(z)+O\left(L z^{24 g}\right)
$$

where $v \asymp 1$ and

$$
W(z)=\prod_{\substack{p \leqslant z \\ p \in \mathcal{B}}}\left(1-\frac{\omega(p)}{p}\right) .
$$

We have $X W(z) \gg x^{\frac{19}{25} \alpha}(\log x)^{-g}$ and $L z^{24 g} \ll x^{2 \varepsilon_{1}+24 g \varepsilon_{3}} \ll X W(z) x^{-\alpha / 5+\eta}$ for any $\eta>0$. Therefore, for $z$ large enough,

$$
S(\mathcal{A}, \mathcal{B}, z) \asymp X W(z) .
$$

To observe that

$$
W(z) \asymp \prod_{\substack{g<p \leqslant x \\ p \nmid a_{1} \cdots a_{r}}}\left(1-\frac{\rho(p)}{p}\right),
$$

which stems from the fact that all fixed prime divisors $p$ of $Q$ are smaller than $g$.

We now expose our proof of Theorem [5. Let $x<n \leqslant x+y$. We write $Q^{*}(n)=$ $p_{1}^{s_{1}} \cdots p_{t}^{s_{t}}$ and define $a_{n}=p_{1}^{s_{1}} \cdots p_{j}^{s_{j}}$ with $j$ maximal so that $a_{n} \leqslant x^{\varepsilon_{1}}$. We let $q_{n}=p_{j+1}$ whenever $j \neq t$, else we let $q_{n}=+\infty$. We thus have a decomposition

$$
Q^{*}(n)=a_{n} b_{n}
$$

with $P^{+}\left(a_{n}\right)<q_{n}$ and $P^{-}\left(b_{n}\right) \geqslant q_{n}$. Accordingly we decompose the $R_{h}(n)$, $1 \leqslant h \leqslant r$, in

$$
R_{h}(n)=a_{h n} b_{h n}
$$


with $P^{+}\left(a_{h n}\right)<q_{n}$ and $P^{-}\left(b_{h n}\right) \geqslant q_{n}$. It follows from the definitions above that $a_{n} \leqslant x^{\varepsilon_{1}}, q_{n}=P^{-}\left(b_{n}\right), a_{n}\left\|Q(n), a_{h n}\right\| R_{h}(n), a_{n}=a_{1 n} \cdots a_{r n}$ and $b_{n}=b_{1 n} \cdots b_{r n}$.

We will distinguish five potentially overlapping classes of integers $x<n \leqslant x+y$ as follows :

$\left(C_{1}\right): a_{n} \leqslant x^{\varepsilon_{1}}, P^{-}\left(b_{n}\right)>x^{\varepsilon_{3}}$,

$\left(C_{2}\right): a_{n} \leqslant x^{\varepsilon_{2}}, P^{-}\left(b_{n}\right) \leqslant x^{\varepsilon_{3}}, b_{n} \neq 1$,

$\left(C_{3}\right): x^{\varepsilon_{2}}<a_{n} \leqslant x^{\varepsilon_{1}}, \omega<P^{+}\left(a_{n}\right) \leqslant x^{\varepsilon_{3}}$

$\left(C_{4}\right): x^{\varepsilon_{2}}<a_{n} \leqslant x^{\varepsilon_{1}}, P^{+}\left(a_{n}\right) \leqslant \omega$,

$\left(C_{5}\right): a_{n} \leqslant x^{\varepsilon_{2}}, b_{n}=1$,

where $\omega$ is a parameter to be chosen later.

For $1 \leqslant i \leqslant 5$ we let

$$
S_{i}=\sum_{n \in\left(C_{i}\right)} F\left(\left|Q_{1}(n)\right|, \ldots,\left|Q_{k}(n)\right|\right)=\sum_{n \in\left(C_{i}\right)} \tilde{F}\left(\left|R_{1}(n)\right|, \ldots,\left|R_{r}(n)\right|\right),
$$

the second equality coming from (2.14).

Contribution of integers $n \in C_{1}$, for which $a_{n} \leqslant x^{\varepsilon_{1}}$ and $P^{-}\left(b_{n}\right)>x^{\varepsilon_{3}}$.

Since $b_{n} \geqslant P^{-}\left(b_{n}\right)^{\Omega\left(b_{n}\right)}$ and $\|Q\| \leqslant x^{\frac{1}{\delta}}$, we have

$$
\Omega\left(b_{n}\right) \leqslant \frac{\log b_{n}}{\log P^{-}\left(b_{n}\right)} \leqslant \frac{\log |Q(n)|}{\log P^{-}\left(b_{n}\right)} \leqslant\left(g+\frac{1}{\delta}\right) \frac{1}{\varepsilon_{3}} .
$$

Therefore by (2.16) we have

$$
\tilde{G}\left(b_{1 n}, \ldots, b_{r n}\right) \leqslant A^{g \Omega\left(b_{n}\right)} \ll 1 .
$$

By 2.15) we then obtain that

$$
S_{1} \ll \sum_{a_{1} \cdots a_{r} \leqslant x^{\varepsilon_{1}}} \tilde{F}\left(a_{1}, \ldots, a_{r}\right) \sum_{\begin{array}{c}
x<n \leqslant x+y \\
a_{h}|| R_{h}(n)(1 \leqslant h \leqslant r) \\
p|Q(n) \Rightarrow p| a_{1} \cdots a_{r} \text { or } p>x^{\varepsilon_{3}}
\end{array}} 1 .
$$

Applying Lemma 6 to bound the inner sum we obtain

$$
S_{1} \ll y \sum_{a_{1} \cdots a_{r} \leqslant x} \tilde{F}\left(a_{1}, \ldots, a_{r}\right) \frac{\hat{\rho}_{\mathbf{R}}\left(a_{1}, \ldots, a_{r}\right)}{\left[a_{1} \kappa\left(a_{1}\right), \ldots, a_{r} \kappa\left(a_{r}\right)\right]} \prod_{\substack{g<p \leqslant x^{\varepsilon_{3}} \\ p \nmid a_{1} \cdots a_{r}}}\left(1-\frac{\rho(p)}{p}\right) .
$$

The inner product is, by (2.3),

$$
\begin{aligned}
& \ll \prod_{h=1}^{r} \prod_{p \mid a_{h}}\left(1-\frac{1}{p}\right)^{-g} \prod_{x^{\varepsilon_{3}<p \leqslant x}}\left(1-\frac{1}{p}\right)^{-g} \prod_{g<p \leqslant x}\left(1-\frac{\rho(p)}{p}\right) \\
& \ll \lambda\left(a_{1}\right) \cdots \lambda\left(a_{r}\right) \prod_{g<p \leqslant x}\left(1-\frac{\rho(p)}{p}\right)
\end{aligned}
$$

where $\lambda(n)=\left(\frac{n}{\varphi(n)}\right)^{g}$. We deduce that

$S_{1} \ll y \prod_{g<p \leqslant x}\left(1-\frac{\rho(p)}{p}\right) \sum_{a_{1} \cdots a_{r} \leqslant x} \tilde{F}\left(a_{1}, \ldots, a_{r}\right) \frac{\hat{\rho}_{\mathbf{R}}\left(a_{1}, \ldots, a_{r}\right)}{\left[a_{1} \kappa\left(a_{1}\right), \ldots, a_{r} \kappa\left(a_{r}\right)\right]} \lambda\left(a_{1}\right) \cdots \lambda\left(a_{r}\right)$.

Applying Lemmas 1, 2 with $\sigma_{h}=1, \theta_{h}=\lambda(1 \leqslant h \leqslant r)$ to the sum over the $a_{i}$ in the above we see that $S_{1}$ is of the expected order of magnitude.

Contribution of integers $n \in C_{2}$, for which $a_{n} \leqslant x^{\varepsilon_{2}}, P^{-}\left(b_{n}\right) \leqslant x^{\varepsilon_{3}}$ and $b_{n} \neq 1$.

Let $q_{n}=P^{-}\left(b_{n}\right)$. By definition of $a_{n}$ we have $a_{n} q_{n}^{e_{n}}>x^{\varepsilon_{1}}$ for some $e_{n} \geqslant 1$. For this $e_{n}$ we have $q_{n}^{e_{n}}>x^{\varepsilon_{1}-\varepsilon_{2}}=x^{2 \varepsilon_{2}}$. We introduce the minimal integer $f_{n}$ such 
that $q_{n}^{f_{n}}>x^{2 \varepsilon_{2}}$. Since $q_{n}^{f_{n}-1} \leqslant x^{2 \varepsilon_{2}}, q_{n}^{f_{n}} \leqslant x^{2 \varepsilon_{2}+\varepsilon_{3}}$ and in particular $f_{n} \leqslant \log x$ and $q_{n}^{f_{n}} \leqslant y$.

By (2.10) and our assumption $\|Q\| \leqslant x^{\frac{1}{\delta}}$ we have

$$
F\left(\left|Q_{1}(n)\right|, \ldots,\left|Q_{k}(n)\right|\right) \leqslant B|Q(n)|^{\varepsilon} \ll x^{\left(g+\frac{1}{\delta}\right) \varepsilon} .
$$

This allows us to bound $S_{2}$ by

$$
S_{2} \ll x^{\left(g+\frac{1}{\delta}\right) \varepsilon} \sum_{f \leqslant \log x} \sum_{\substack{q \leqslant x^{\varepsilon} \\ x^{2 \varepsilon_{2}}<q^{f} \leqslant y}} \sum_{\substack{x<n \leqslant x+y \\ q^{f} \mid Q^{*}(n)}} 1 .
$$

The innerest sum is

$$
\leqslant \rho^{*}\left(q^{f}\right)\left(\frac{y}{q^{f}}+1\right) \ll y \frac{\rho^{*}\left(q^{f}\right)}{q^{f}} \ll y q^{-\frac{f}{g}}
$$

by (2.6). Therefore

$$
S_{2} \ll y x^{\left(g+\frac{1}{\delta}\right) \varepsilon} \sum_{f \leqslant \log x} \sum_{\substack{q \leqslant x^{\varepsilon_{3}} \\ x^{-2 \varepsilon_{2}}<q^{f}}} q^{-\frac{f}{g}} \ll y x^{\left(g+\frac{1}{\delta}\right) \varepsilon+\varepsilon_{3}-2 \frac{\varepsilon_{2}}{g}} \ll y x^{-c} \log x
$$

with $c=\frac{\alpha}{25 g}$. This is readily seen to be lower than the expected order of magnitude since the right-hand side of (3.1) is $\gg y(\log x)^{-g}$. This last fact follows from our assumption $F(1, \ldots, 1)=1$ and (2.3).

Contribution of integers $n \in C_{3}$, for which $x^{\varepsilon_{2}}<a_{n} \leqslant x^{\varepsilon_{1}}$ and $\omega<P^{+}\left(a_{n}\right) \leqslant$ $x^{\varepsilon_{3}}$.

We define $\ell_{n}:=P^{+}\left(a_{n}\right)$. We write $a_{n}=\ell_{n}^{\nu_{n}} c_{n}$ with $\ell_{n} \nmid c_{n}$ and $a_{n h}=\ell_{n}^{\nu_{h n}} c_{h n}$ with $\ell_{n} \nmid c_{h n}(1 \leqslant h \leqslant r)$. By (2.17), we have

$$
\tilde{G}\left(\ell_{n}^{\nu_{1 n}}, \ldots, \ell_{n}^{\nu_{r n}}\right) \leqslant D\left(\ell_{n}^{\nu_{n}}\right)
$$

where $D$ is the multiplicative function defined by

$$
D\left(p^{\nu}\right)=\min \left(A^{g \nu}, B p^{g \varepsilon \nu}\right) \quad(\nu \geqslant 1)
$$

for primes $p$. We also have, as in the case of the class $\left(C_{2}\right)$,

$$
\Omega\left(b_{n}\right) \leqslant\left(g+\frac{1}{\delta}\right) \frac{\log x}{\log \ell_{n}}
$$

and therefore, upon using (2.16),

$$
\tilde{G}\left(b_{1 n}, \ldots, b_{r n}\right) \leqslant A^{g \Omega\left(b_{n}\right)} \leqslant e^{L u_{n}}
$$

with $u_{n}:=\frac{\log x}{\log \ell_{n}}$ and $L:=g\left(g+\frac{1}{\delta}\right) \log A$. Note that $L$ depends on the usual parameters.

Applying (2.15), we then obtain

$$
S_{3} \ll \sum_{\substack{\ell^{\nu} \leqslant x^{\varepsilon_{1}} \\
w<\ell \leqslant x^{\varepsilon_{3}}}} D\left(\ell^{\nu}\right) e^{L u} \sum_{\substack{\frac{x^{\varepsilon_{2}}}{\ell^{\nu}} \leqslant c_{1} \cdots c_{r} \leqslant \frac{x^{\varepsilon_{1}}}{\ell^{\nu}} \\
P^{+}\left(c_{1} \cdots c_{r}\right)<\ell}} \tilde{F}\left(c_{1}, \ldots, c_{r}\right) \sum_{\begin{array}{c}
x<n \leqslant x+y \\
c_{h} \| R_{h}(n)(1 \leqslant h \leqslant r) \\
\ell^{\nu} \mid Q^{*}(n) \\
p|Q(n) \Rightarrow p| \ell_{1} \cdots c_{r} \text { or } p>x^{\varepsilon_{3}}
\end{array}} 1
$$

where $u=\frac{\log x}{\log \ell}$. Now Lemma 6 can easily be modified to bound the inner sum above, the new condition $\ell^{\nu} \mid Q^{*}(n)$ changing the right-hand side of (5.2) upto a 
factor $\frac{\rho^{*}\left(\ell^{\nu}\right)}{\ell^{\nu}}$. We thereby obtain

$$
\begin{aligned}
S_{3} \ll y & \sum_{w<\ell \leqslant x} \sum_{\nu} D\left(\ell^{\nu}\right) \frac{\rho^{*}\left(\ell^{\nu}\right)}{\ell^{\nu}} e^{L u} \\
& \times \sum_{\substack{\frac{x^{\varepsilon}}{\ell_{2}} \\
P^{+}\left(c_{1} \cdots c_{r}\right)<c_{r}}} \tilde{F}\left(c_{1}, \ldots, c_{r}\right) \frac{\hat{\rho}_{\mathbf{R}}\left(c_{1}, \ldots, c_{r}\right)}{\left[c_{1} \kappa\left(c_{1}\right), \ldots, c_{r} \kappa\left(c_{r}\right)\right]} \prod_{\substack{g<p \leqslant q \\
p \nmid \ell c_{1} \cdots c_{r}}}\left(1-\frac{\rho(p)}{p}\right) .
\end{aligned}
$$

The inner product is, by (2.3),

$$
\begin{aligned}
& \ll \prod_{h=1}^{r} \prod_{p \mid c_{h}}\left(1-\frac{1}{p}\right)^{-g} \prod_{q<p \leqslant x}\left(1-\frac{1}{p}\right)^{-g} \prod_{g<p \leqslant x}\left(1-\frac{\rho(p)}{p}\right) \\
& \ll \lambda\left(c_{1}\right) \cdots \lambda\left(c_{r}\right) u^{g} \prod_{g<p \leqslant x}\left(1-\frac{\rho(p)}{p}\right)
\end{aligned}
$$

where $\lambda(n)=\left(\frac{n}{\varphi(n)}\right)^{g}$. Letting $\chi=\frac{3 L}{\varepsilon_{2}}$ and $\beta=\frac{\chi}{\log \ell}$, we therefore have

$$
\begin{aligned}
& S_{3} \ll y \prod_{g<p \leqslant x}\left(1-\frac{\rho(p)}{p}\right) \sum_{w<\ell \leqslant x} \sum_{\nu} D\left(\ell^{\nu}\right) \frac{\rho^{*}\left(\ell^{\nu}\right)}{\ell^{\nu}} e^{L u} u^{g}\left(\frac{x^{\varepsilon_{2}}}{\ell^{\nu}}\right)^{-\beta} \\
& \times \sum_{P^{+}\left(c_{1} \cdots c_{r}\right)<\ell} F\left(c_{1}, \ldots, c_{r}\right) \frac{\hat{\rho}_{\mathbf{R}}\left(c_{1}, \ldots, c_{r}\right)}{\left[c_{1} \kappa\left(c_{1}\right), \ldots, c_{r} \kappa\left(c_{r}\right)\right]} \lambda\left(c_{1}\right) \cdots \lambda\left(c_{r}\right)\left(c_{1} \cdots c_{r}\right)^{\beta} .
\end{aligned}
$$

We now remark that

$$
e^{L u} u^{g}\left(\frac{x^{\varepsilon_{2}}}{\ell^{\nu}}\right)^{-\beta}=e^{-2 L u} u^{g} e^{\nu \chi} \ll e^{-L u} e^{\nu \chi},
$$

and we apply Lemmas 1, 3, 5 with $\sigma_{h}=\lambda(1 \leqslant h \leqslant r)$ to the sum over the $c_{i}$ in (5.4) to obtain

$$
\begin{aligned}
S_{3} \ll y \prod_{g<p \leqslant x}(1- & \left.\frac{\rho(p)}{p}\right)\left(\sum_{w<\ell \leqslant x} e^{-L u} \sum_{\nu} e^{\nu \chi} D\left(\ell^{\nu}\right) \frac{\rho^{*}\left(\ell^{\nu}\right)}{\ell^{\nu}}\right) \\
& \times \sum_{c_{1} \cdots c_{r} \leqslant x} \tilde{F}\left(c_{1}, \ldots, c_{r}\right) \frac{\hat{\rho}_{\mathbf{R}}\left(c_{1}, \ldots, c_{r}\right)}{\left[c_{1} \kappa\left(c_{1}\right), \ldots, c_{r} \kappa\left(c_{r}\right)\right]} \lambda\left(c_{1}\right) \cdots \lambda\left(c_{r}\right) .
\end{aligned}
$$

Using (2.4) and (2.6) we see that, taking $\omega=e^{2 g \chi}$,

$$
\sum_{\nu} e^{\nu \chi} D\left(\ell^{\nu}\right) \frac{\rho^{*}\left(\ell^{\nu}\right)}{\ell^{\nu}} \ll \frac{1}{\ell}+\sum_{\nu>2 g} e^{\nu \chi} \ell^{g \varepsilon \nu} \ell^{-\frac{\nu}{g}} \ll \frac{1}{\ell} .
$$

Also by integration by parts we have

$$
\sum_{\ell \leqslant x} \frac{e^{-L u}}{\ell} \ll 1 .
$$

The sum over $\ell$ in (5.5) is therefore bounded. Applying Lemmas 1, 2 with $\sigma_{h}=1$, $\theta_{h}=\lambda(1 \leqslant h \leqslant r)$ to the sum over the $c_{i}$ in (5.5) we thus see that (5.5) is compatible with (3.1).

Contribution of integers $n \in C_{4}$, for which $x^{\varepsilon_{2}}<a_{n} \leqslant x^{\varepsilon_{1}}$ and $P^{+}\left(a_{n}\right) \leqslant \omega$. 
We use the trivial bound (5.3) to obtain

$$
\begin{aligned}
& S_{4} \ll x^{\left(g+\frac{1}{\delta}\right) \varepsilon} \sum_{\substack{x^{\varepsilon_{2}}<a \leqslant x^{\varepsilon_{1}} \\
P^{+}(a) \leqslant \omega}} \sum_{\substack{x<n \leqslant x+y \\
a \mid Q^{*}(n)}} 1
\end{aligned}
$$

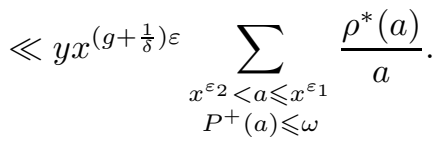

For integers $a$ such that $P^{+}(a) \leqslant \omega$ we have $\omega(a) \leqslant \pi(\omega) \ll 1$ and hence, by (2.6), $\rho^{*}(a) \leqslant g^{\omega(a)} a^{1-\frac{1}{g}} \ll a^{1-\frac{1}{g}}$. Inserting this bound in (5.6) we obtain

$$
S_{4} \ll y x^{\left(g+\frac{1}{\delta}\right) \varepsilon} \sum_{\substack{x^{\varepsilon_{2}}<a \leqslant x_{1} \\ P^{+}(a) \leqslant \omega}} a^{-\frac{1}{g}} \ll y x^{\left(g+\frac{1}{\delta}\right) \varepsilon-\frac{\varepsilon_{2}}{g}}(\log x)^{\omega} \ll y x^{-c}(\log x)^{\omega}
$$

with $c=\frac{\alpha}{25 g}$. This is compatible with (3.1) as argued in the case of integers $n \in\left(C_{2}\right)$.

Contribution of integers $n \in C_{5}$, for which $a_{n} \leqslant x^{\varepsilon_{2}}$ and $b_{n}=1$.

We use the trival bound (5.3) to obtain

$$
S_{5} \ll x^{\left(g+\frac{1}{\delta}\right) \varepsilon} \sum_{a \leqslant x^{\varepsilon_{2}}} \sum_{\substack{x<n \leqslant x+y \\ Q^{*}(n)=a}} 1 \ll x^{\left(g+\frac{1}{\delta}\right) \varepsilon+\varepsilon_{2}} \ll y x^{-c} .
$$

with $c=-\frac{47}{50} \alpha$. This is compatible with (3.1) as argued in the case of integers $n \in\left(C_{2}\right)$.

\section{Proof of Corollaries 1 and 2}

To derive Corollaries 1 and 2 from Theorem 5 we focus on the sum

$$
\tilde{S}=\sum_{n_{1} \cdots n_{r} \leqslant x} \tilde{F}\left(n_{1}, \ldots, n_{r}\right) \frac{\hat{\rho}_{\mathbf{R}}\left(n_{1}, \ldots, n_{r}\right)}{\left[n_{1} \kappa\left(n_{1}\right), \ldots, n_{r} \kappa\left(n_{r}\right)\right]}
$$

appearing in the right-hand side of (3.1). We shall establish upper bounds for $\tilde{S}$ as well as lower bounds that we need for the proof of Theorem [6. Corollary 1 is a direct consequence of the following Lemma.

Lemma 7. We have

$$
\tilde{S} \ll \Delta_{D^{*}} \sum_{\substack{a_{1} \cdots a_{r} \leqslant x \\\left(a_{1} \cdots a_{r}, D^{*}\right)=1 \\\left(a_{i}, a_{j}\right)=1(i \neq j)}} \tilde{F}\left(a_{1}, \ldots, a_{r}\right) \frac{\rho_{R_{1}}\left(a_{1}\right) \cdots \rho_{R_{r}}\left(a_{r}\right)}{a_{1} \cdots a_{r}} .
$$

Proof. For all $1 \leqslant h \leqslant r$, we write $n_{h}=d_{h} a_{h}$ with $d_{h} \mid D^{* \infty}$ and $\left(a_{h}, D^{*}\right)=1$. By (2.15) and the submultiplicativity of $\tilde{G}$, we then have

$$
\tilde{S} \ll \Delta_{4} \sum_{\substack{a_{1} \cdots a_{r} \leqslant x \\\left(a_{1} \cdots a_{r}, D^{*}\right)=1}} \tilde{F}\left(a_{1}, \ldots, a_{r}\right) \frac{\hat{\rho}_{\mathbf{R}}\left(a_{1}, \ldots, a_{r}\right)}{\left[a_{1} \kappa\left(a_{1}\right), \ldots, a_{r} \kappa\left(a_{r}\right)\right]}
$$

where

$$
\Delta_{4}=\prod_{p \mid D^{*}}\left(1+\sum_{\nu_{1}, \ldots, \nu_{r}}^{\prime} \tilde{G}\left(p^{\nu_{1}}, \ldots, p^{\nu_{r}}\right) \frac{\hat{\rho}_{\mathbf{R}}\left(p^{\nu_{1}}, \ldots, p^{\nu_{r}}\right)}{p^{\max _{h}\left(\nu_{h}\right)+1}}\right)
$$


Now let $1 \leqslant m \leqslant r$ and define $\mu_{m}=\operatorname{deg}\left(R_{m}\right)$. By the definition (2.8) of $\hat{\rho}_{\mathbf{R}}$ and (2.7), we have

$$
\frac{\hat{\rho}_{\mathbf{R}}\left(p^{\nu_{1}}, \ldots, p^{\nu_{r}}\right)}{p^{\max _{h}\left(\nu_{h}\right)}} \leqslant \frac{\rho_{R_{m}}\left(p^{\nu_{m}}\right)}{p^{\nu_{m}}} \leqslant \mu_{m} p^{-\nu_{m} / \mu_{m}} .
$$

Using this bound and (2.16), we obtain

$$
\sum_{\substack{\nu_{m}>\mu_{m} \\ \nu_{1}+\cdots+\nu_{r} \leqslant 2 g}} \tilde{G}\left(p^{\nu_{1}}, \ldots, p^{\nu_{r}}\right) \frac{\hat{\rho}_{\mathbf{R}}\left(p^{\nu_{1}}, \ldots, p^{\nu_{r}}\right)}{p^{\max _{h}\left(\nu_{h}\right)+1}} \ll \sum_{\nu>\mu_{m}} p^{-\nu / \mu_{m}} \ll \frac{1}{p^{1+1 / \nu_{m}}} .
$$

Since this is true for all $1 \leqslant m \leqslant r$ and since a similar bound holds for the tail $\sum_{\nu_{1}+\cdots+\nu_{r}>2 g}$ by (4.4), it follows that $\Delta_{4} \asymp \Delta_{D^{*}}$, where $\Delta_{D^{*}}$ is defined by (3.2).

It remains to rewrite the sum over the $a_{i}$ in (6.2). To this end we use certain algebraic facts about the discriminant and the resultant, the proof of which can be found in e.g. [7]. For $h \neq i$ there exists polynomials $U, V$ in $\mathbb{Z}[X]$ such that

$$
R_{h}(X) U(X)+R_{i}(X) V(X)=\operatorname{Res}\left(R_{h}, R_{i}\right)
$$

where $\operatorname{Res}\left(R_{h}, R_{i}\right)$ is the resultant of $R_{h}$ and $R_{i}$. When $\hat{\rho}_{\mathbf{R}}\left(a_{1}, \ldots, a_{r}\right)$ is non-zero there exists an integer $n$ such that $a_{i} \mid R_{i}(n)$ and $a_{j} \mid R_{j}(n)$. Taking $X=n$ in (6.4) we then see that $\left(a_{i}, a_{h}\right) \mid \operatorname{Res}\left(R_{h}, R_{i}\right)$. Since $\operatorname{Disc}\left(R_{h} R_{i}\right)=\operatorname{Res}\left(R_{h}, R_{i}\right)^{2} \operatorname{Disc}\left(R_{h}\right) \operatorname{Disc}\left(R_{i}\right)$ and $\operatorname{Disc}\left(R_{h} R_{i}\right) \mid \operatorname{Disc}\left(Q^{*}\right)=D^{*}$, we have that $\operatorname{Res}\left(R_{h}, R_{i}\right) \mid D^{*}$. Therefore $\left(a_{i}, a_{h}\right) \mid D^{*}$, and since the $a_{j}$ are coprime to $D^{*}$ we have further $\left(a_{i}, a_{h}\right)=1$. We deduce that $\hat{\rho}_{\mathbf{R}}\left(a_{1}, \ldots, a_{r}\right)$ is zero unless the $a_{i}$ are mutually coprime in which case we have, by multiplicativity of $\hat{\rho}_{\mathbf{R}}$,

$$
\begin{aligned}
\hat{\rho}_{\mathbf{R}}\left(a_{1}, \ldots, a_{r}\right) & =\prod_{h=1}^{r} \hat{\rho}_{\mathbf{R}}^{(h)}\left(a_{h}\right) \\
& =\prod_{h=1}^{r} \prod_{p^{\nu} \| a_{h}}\left(\rho_{R_{h}}\left(p^{\nu_{h}}\right) p-\rho_{R_{h}}\left(p^{\nu_{h}+1}\right)\right) \\
& \leqslant \prod_{h=1}^{r} \rho_{R_{h}}\left(a_{h}\right) \kappa\left(a_{h}\right) .
\end{aligned}
$$

Inserting this back in (6.2) we recover (6.1).

Corollary 2 is obtained in a similar fashion, by applying the following Lemma.

Lemma 8. We have

$$
\tilde{S} \ll \Delta_{D^{*}} \prod_{\substack{p \leqslant x \\ p \nmid D^{*}}} \prod_{h=1}^{r}\left(1+\tilde{G}^{(h)}(p) \frac{\rho_{R_{h}}(p)}{p}\right) .
$$

The right-hand side in the above is also a lower bound for $\tilde{S}$ when $F$ is assumed to be multiplicative.

Proof. Applying Lemmas 1, 4, 5ith $\sigma_{h}=1(1 \leqslant h \leqslant r)$, we obtain

$$
\tilde{S} \asymp \sum_{P^{+}\left(n_{1} \cdots n_{r}\right) \leqslant x^{(2 g-2) / \delta}} \tilde{F}\left(n_{1}, \ldots, n_{r}\right) \frac{\hat{\rho}_{\mathbf{R}}\left(n_{1}, \ldots, n_{r}\right)}{\left[n_{1} \kappa\left(n_{1}\right), \ldots, n_{r} \kappa\left(n_{r}\right)\right]} .
$$

We have $D^{*} \ll\left\|Q^{*}\right\|^{2 g-2}$. Since $Q^{*} \mid Q$, we have $\left\|Q^{*}\right\| \leqslant C\|Q\|$ where $C$ depends on $g$ at most (see e.g. [11] for precise results on the norm of a factor of a polynomial). By our assumption $x \geqslant\|Q\|^{\delta}$ we therefore have $D^{*} \leqslant x^{(2 g-2) / \delta}$ for $x$ large 
enough with respect to the usual parameters. Using this fact and $\tilde{F} \leqslant \tilde{G}$ and the submultiplicativity of $\tilde{G}$ we can write

$$
\tilde{S} \ll \Delta_{4} \prod_{\substack{p \leqslant x^{(2 g-2) / \delta} \\ p \nmid D^{*}}}\left(1+\sum_{\nu_{1}, \ldots, \nu_{r}}^{\prime} \tilde{G}\left(p^{\nu_{1}}, \ldots, p^{\nu_{r}}\right) \frac{\hat{\rho}_{\mathbf{R}}\left(p^{\nu_{1}}, \ldots, p^{\nu_{r}}\right)}{p^{\max _{h}\left(\nu_{h}\right)+1}}\right) .
$$

where $\Delta_{4}$ is defined by (6.3) as previously, and has been proven to be $\asymp \Delta_{D^{*}}$. When $F$ is multiplicative, so is $\tilde{F}=\tilde{G}$, and the right-hand side of (6.5) is therefore also a lower bound for $\tilde{S}$.

Now by (4.3) the main term of the product in (6.5) is $1+O\left(\frac{1}{p}\right)$ and we can thus restrict the product to primes $p \leqslant x$. By (4.4) we can also restrict the inner sum in (6.5) to variables $\nu_{i}$ satisfying $\nu_{1}+\cdots+\nu_{r} \leqslant 2 g$. For those values we have, by (2.9), (2.5) and (2.16),

$$
\tilde{G}\left(p^{\nu_{1}}, \ldots, p^{\nu_{r}}\right) \frac{\hat{\rho}_{\mathbf{R}}\left(p^{\nu_{1}}, \ldots, p^{\nu_{r}}\right)}{p^{\max _{h}\left(\nu_{h}\right)+1}} \leqslant A^{2 g^{2}} \frac{g^{*}}{p^{\nu_{1}+\cdots+\nu_{r}}} .
$$

We can therefore further restrict the inner sum in (6.5) to variables $\nu_{i}$ satisfying the condition $\nu_{1}+\cdots+\nu_{r} \leqslant 1$. The Lemma then easily follows.

\section{Proof of Theorem 6}

The purpose of this section is to prove Theorem 6. The upper bounds follow immediately from Theorem 5 and Corollary 2, we are therefore only concerned with proving the lower bounds.

In this section we assume that the requirements of Theorem 6 are fullfilled. We also now allow implicit constants to depend on the paramater $\eta<1$ on top of the usual parameters. We retain the definitions (5.1) of $\varepsilon_{1}, \varepsilon_{2}$ and $\varepsilon_{3}$.

We let

$$
S=\sum_{x<n \leqslant x+y} F\left(\left|Q_{1}(n)\right|, \ldots,\left|Q_{k}(n)\right|\right) .
$$

For an integer $n$ we write

$$
Q(n)=a_{n} b_{n}, \quad R_{h}(n)=a_{h n} b_{h n} \quad(1 \leqslant h \leqslant r)
$$

with $P^{+}\left(a_{n}\right)<x^{\varepsilon_{3}}, P^{-}\left(b_{n}\right) \geqslant x^{\varepsilon_{3}}, P^{+}\left(a_{h n}\right)<x^{\varepsilon_{3}}$ and $P^{-}\left(b_{h n}\right) \geqslant x^{\varepsilon_{3}}$.

Since $b_{n} \geqslant P^{-}\left(b_{n}\right)^{\Omega\left(b_{n}\right)}$ and $\|Q\| \leqslant x^{\frac{1}{\delta}}$ we have

$$
\Omega\left(b_{n}\right) \leqslant \frac{\log b_{n}}{\log P^{-}\left(b_{n}\right)} \leqslant \frac{\log |Q(n)|}{\log P^{-}\left(b_{n}\right)} \leqslant\left(g+\frac{1}{\delta}\right) \frac{1}{\varepsilon_{3}} .
$$

By (3.3) we then have

$$
\tilde{F}\left(b_{1 n}, \ldots, b_{r n}\right) \geqslant \eta^{\Omega\left(b_{n}\right)} \gg 1 .
$$

Keeping only the integers $n$ such that $a_{1 n} \cdots a_{r n} \leqslant x^{\varepsilon_{1}}$, we obtain, by multiplicativity of $F$ and the above bound,

$$
S \gg \sum_{a_{1} \cdots a_{r} \leqslant x^{\varepsilon_{1}}} \tilde{F}\left(a_{1}, \ldots, a_{r}\right) \sum_{\begin{array}{c}
x<n \leqslant x+y \\
a_{h} \| R_{h}(n)(1 \leqslant h \leqslant r) \\
p|Q(n) \Rightarrow p| a_{1} \cdots a_{r} \text { or } p>x^{\varepsilon_{3}}
\end{array}} 1 .
$$


The inner sum can be estimated by applying Lemma [ ] using the fact that $Q$ has no fixed prime divisor. This yields, as in the proof of Theorem 5 ,

$S \gg y \prod_{g<p \leqslant x}\left(1-\frac{\rho(p)}{p}\right) \sum_{a_{1} \cdots a_{r} \leqslant x^{\varepsilon_{1}}} \tilde{F}\left(a_{1}, \ldots, a_{r}\right) \frac{\hat{\rho}_{\mathbf{R}}\left(a_{1}, \ldots, a_{r}\right)}{\left[a_{1} \kappa\left(a_{1}\right), \ldots, a_{r} \kappa\left(a_{r}\right)\right]} \lambda\left(a_{1}\right) \ldots \lambda\left(a_{r}\right)$

where $\lambda(n)=\left(\frac{\varphi(n)}{n}\right)^{g}$. Applying Lemmas 1, 2 with $\sigma_{h}=\lambda, \theta_{h}=\lambda^{-1}(1 \leqslant h \leqslant r)$ to the sum over the $a_{i}$ in the above we obtain

$$
S \gg y \prod_{g<p \leqslant x}\left(1-\frac{\rho(p)}{p}\right) \sum_{a_{1} \cdots a_{r} \leqslant x^{\varepsilon}} \tilde{F}\left(a_{1}, \ldots, a_{r}\right) \frac{\hat{\rho}_{\mathbf{R}}\left(a_{1}, \ldots, a_{r}\right)}{\left[a_{1} \kappa\left(a_{1}\right), \ldots, a_{r} \kappa\left(a_{r}\right)\right]} .
$$

Further applying Lemmas 1, 4, 5 with $\sigma_{h}=1$ to the sum over the $a_{i}$ we recover the lower bound in (3.4). The lower bound in (3.5) then follows from Lemma 8 .

\section{REFERENCES}

[1] R. de la Bretèche and T. D. Browning, Le problème des diviseurs pour des formes binaires de degré 4. To appear in J. Reine Angew. Math. (2008).

[2] R. de la Bretèche and T.B. Browning, Sums of arithmetic functions over values of binary forms. Acta Arith. 125 (2007), 291-304.

[3] S. Daniel, Uniform bounds for short sums of certain arithmetic functions of polynomial arguments. Unpublished manuscript.

[4] H. Halberstam and H.-E. Richert, Sieve methods. (Academic Press, 1974).

[5] R. Holowinsky, Sieving for Mass equidistribution. Ann. of Math. (2) 172 (2010), 1499-1516.

[6] R. Holowinsky and K. Soundararajan, Mass equidistribution for Hecke eigenforms. Ann. of Math. (2) 172 (2010), 1517-1528.

[7] S. Lang, Algebra. (Spinger-Verlag, 2002).

[8] T. Nagell, Introduction to number theory. (Wiley, 1951).

[9] M. Nair, Multiplicative functions of polynomial values in short intervals. Acta Arith. 62 (1992), 257-269.

[10] M. Nair and G. Tenenbaum, Short sums of certain arithmetic functions. Acta Math. 180 (1998), 119-144.

[11] I. E. Pritsker, An inequality for the norm of a polynomial factor. Proc. Amer. Math. Soc. 129 (2001), 2283-2291.

[12] P. Shiu, A Brun-Titschmarsh theorem for multiplicative functions. J. Reine Angew. Math. 313 (1980), 161-170.

[13] C. L. Stewart, On the number of solutions of polynomial congruences and Thue equations. J. Amer. Math. Soc. 4 (1991), 793-835.

Kevin Henriot

Université de Montréal

Département de Mathématiques et de Statistique,

Pavillon André-Aisenstadt, bureau 5190,

2900 Édouard-Montpetit

Montréal, Québec, Canada, H3C 3J7

email: henriot@dms.umontreal.ca 